\title{
Zusatznutzen für neue Dauertherapie bestätigt
}

_ Spiolto $^{\circledast}$ (Tiotropium/Olodaterol) Respimat $^{\oplus}$ ist seit Juli 2015 als neue Dauertherapie für die chronisch obstruktive Lungenerkrankung (COPD) zugelassen. Im Rahmen der frühen Nutzenbewertung hat der Gemeinsame Bundesausschuss (G-BA) seinen Beschluss zu Spiolto $^{\circledast}$ Respimat $^{\oplus}$ vorgelegt. Darin bescheinigt der G-BA Spiolto ${ }^{\circledR}$ Respimat $^{\circledR}$ einen Zusatznutzen im Vergleich zu Spiriva ${ }^{\oplus}$ Respimat $^{\circledast}$ als langwirksame Dauertherapie zur einmal täglichen Inhalation für COPD-Patienten.

Spiolto $^{\circledR}$ Respimat $^{\circledR}$ basiert auf Tiotropium, dem aktiven Wirkstoff in Spiriva ${ }^{\oplus}$, und wird durch den schnell und ultralang wirksamen $\beta_{2}$-Agonisten (LABA) Olodaterol zusätzlich verbessert. Die neue COPD-Dauertherapie ist eine vielversprechende Option für erwachsene
Patienten mit COPD aller Schweregrade, gerade für die frühzeitige therapeutische Intervention ab dem Start der Dauertherapie (ab GOLD-Stadium II).

Spiolto ${ }^{\oplus}$ Respimat $^{\oplus}$ führt zu einer signifikanten Verbesserung der Lungenfunktion, der COPD-Symptomatik (Atemnot/Dyspnoe), der Lebensqualität sowie einem geringeren Verbrauch an Notfallmedikation im Vergleich zu Spiriva ${ }^{\circledast}$ Respimat ${ }^{\circledR}$.

Die Verbesserung der Lungenfunktion (gemessen als Trough $\mathrm{FEV}_{1}$ vs. Ausgangswert) bei Patienten ohne vorherige Dauertherapie war mehr als doppelt so groß wie diejenige unter einer Monotherapie mit Spiriva ${ }^{\oplus}$ Respimat $^{\oplus}$ bei Patienten im GOLD-Stadium II.

Red.

- Nach Informationen von Boehringer Ingelheim

\section{Kurz notiert}

Nächtliche Wadenkrämpfe bei Erwachsenen $\rightarrow$ Wenn Wadenkrämpfe sehr häufig nachts auftreten und besonders schmerzhaft sind, vermindern sie erheblich die Lebensqualität. Betroffene sind in ihrer Alltagsfunktionalität stark eingeschränkt. Viele Patienten klagen über Schlafprobleme und fühlen sich ständig übermüdet. Auch können noch Stunden nach dem Krampfereignis Schmerzen im betroffenen Muskelbereich bestehen.

Die therapeutische Intervention erfordert eine rasche Muskelkrampflösung und Schmerzlinderung sowie eine wirksame Vorbeugung - auch ohne dass der Auslöser bekannt wäre. Diesen Anspruch erfüllt die Behandlung mit Chininsulfat. Auf die Wirksamkeit der Substanz bei Wadenkrämpfen wird in der S1-Leitlinie der Deutschen Gesellschaft für Neurologie explizit hingewiesen. Als einziges chininsulfathaltiges Arzneimittel ist in Deutschland Limptar ${ }^{\circledast} \mathrm{N}$ zugelassen. - Red.

- Deutsche Gesellschaft für Neurologie (DGN). S1-Leitlinie "Crampi/Muskelkrampf". AWMF-Registernr. 030/037 (Stand: September 2012)

\section{Hepatitis C}

\section{Hohe Heilungschancen für Opioidabhängige}

_ In den meisten westlichen Ländern gilt intravenöser Drogenkonsum als Hauptrisikofaktor für eine Infektion mit dem Hepatitis-C-Virus (HCV). Dennoch ist diese Patientengruppe in Studien unterrepräsentiert, berichtete Prof. Gregory Dore, Sydney. Dore stellte eine erste große, randomisierte, doppelblinde, placebokontrollierte Studie vor, an der 301 opioidsubstituierte, therapienaive HCV-Patienten mit den VirusGenotypen (GT) 1, 4 und 6 teilnahmen [Dore G et al. Hepatology 2015, 227A. Abstract 40]. 76,1\% waren mit GT 1a, $15 \%$ mit GT 1b, 6\% mit GT 4 und 3\% mit GT 6 infiziert. Rund 20\% der Patienten hatten bereits eine Zirrhose und 7\% eine HIV-Koinfektion.

Die Patienten erhielten für 12 Wochen verblindet entweder sofort Grazoprevi/ Elbasvir $(n=201)$ oder Placebo $(n=100)$. Die Placebo-Patienten erhielten danach offen für 12 Wochen Grazoprevir/Elbasvir. Grazoprevir ist ein NS3/4A-Inhibitor und Elbasvir ein NS5A-Inhibitor. Beide Substanzen sind in einer Tablette kombiniert (100 mg/50 mg).

\section{Anhaltendes virologisches Ansprechen bei über $91,5 \%$}

Mit Grazoprevir/Elbasvir erreichten 91,5\% der Patienten eine SVR12, sprachen also auch in der zwölften Woche nach Ende der Therapie virologisch anhaltend an (sustained virological response, SVR) und waren damit geheilt. Die SVR12-Rate beim GT la betrug 96\%, beim GT 1b 97\%, beim GT $4100 \%$ und beim GT 6 60\%. Insgesamt erlitten sieben Patienten einen Relapse. Eine Reinfektion wurde bei fünf Patienten nachgewiesen.

Dore betonte, dass „die Adhärenz trotz des Beigebrauchs von Drogen ausgezeichnet war". In der verblindeten Phase nahmen im Grazoprevir/Elbasvir-Arm 100\% der Patienten mindestens $80 \%$ aller Tabletten ein, 99\% mindestens mehr als 90\%

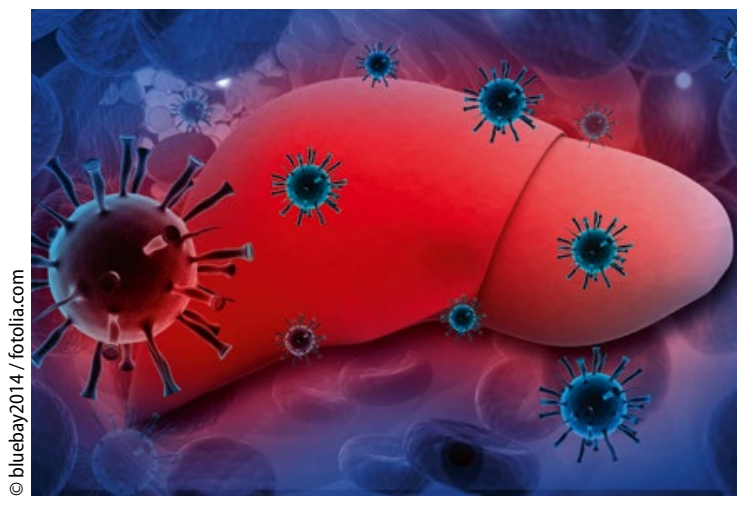

und 96,5\% mehr als 95\%. Die Adhärenz im Placeboarm war vergleichbar gut.

Die Therapie mit Elbasvir/Grazoprevir sei auch in dieser Patientenpopulation gut verträglich, so Dore. Die häufigsten unerwünschten Ereignisse unter Verum im Vergleich zu Placebo waren Müdigkeit (16\% vs. 20\%), Kopfschmerzen (13\% vs. $14 \%)$, Übelkeit (11\% vs. 9\%) und Durchfall (10\% vs. 9\%). In der Placebogruppe gab es einen Todesfall.

Andrea Warparkowski

- Referat im Rahmen des Kongresses der American Association for the Study of Liver Diseases (AASLD); San Francisco, 13.-17. November 2015 (unterstützt von MSD) 